\title{
Digoxin as a Treatment for Patients With Idiopathic Intracranial Hypertension
}

Jen Haagensen, MS

Carl Hoegerl, DO, MSc

From Geisinger Medical Center in Danville,

Pennsylvania.

Financial Disclosures:

None reported.

Address correspondence to Carl Hoegerl, DO, MSc, Liberty University College of Osteopathic Medicine,

1971 University Blvd, Lynchburg, VA 24515-0001.

E-mail: hoegerl@yahoo.com

Submitted

December 27, 2012;

final revision received

August 14, 2013;

accepted

August 29, 2013.
Idiopathic intracranial hypertension (IIH)_sometimes called pseudotumor cerebri-is a neurologic condition distinguished by any of the following symptoms: headache, increased cerebrospinal fluid pressure, papilledema, vision loss, diplopia, tinnitus, deafness, nausea and vomiting, or sixth nerve palsy. Medical and surgical management options are available for patients with IIH. The authors report a case of IIH that was successfully resolved with digoxin after standard avenues of therapy were exhausted.

J Am Osteopath Assoc. 2014;114(3):213-215

doi:10.7556/jaoa.2014.039

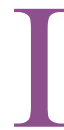
diopathic intracranial hypertension (IIH) — also known as pseudotumor cerebriis a neurologic condition that can manifest as headache, increased cerebrospinal fluid (CSF) pressure, papilledema, vision loss, diplopia, tinnitus, deafness, nausea and vomiting, or sixth nerve palsy. Risk factors include female sex, weight gain, obesity, certain medications (eg, growth hormone, minocycline, doxycycline ${ }^{1}$ ), problems with metabolizing vitamin A supplements, and rheumatologic disorders (eg, lupus). The condition is difficult to diagnose because it may involve specialist consultations (eg, neurology, ophthalmology) or procedures (eg, lumbar puncture). Neuroimaging studies do not show evidence of hydrocephalus, masses, or vascular or structural abnormalities. Cerebrospinal fluid composition is usually normal. ${ }^{2}$ At a rate of 7.9 per 100,000 , obese females of childbearing age are disproportionately affected by $\mathrm{IIH}^{3}$

The pathophysiologic process of IIH is uncertain. One theory posits that there is resistance of CSF outflow at the level of the arachnoid granulations. Other theories attribute the condition to abnormalities of the cerebral venous outflow or a change in sodium and water retention mechanisms. ${ }^{4}$ Another cause may be problems with vitamin A metabolism ${ }^{5}$ or obesity-related increased abdominal pressure and intracranial venous pressure. ${ }^{5,6}$

Management of IIH involves both medical and surgical modalities. If a patient is obese, weight loss is encouraged, and acetazolamide and topiramate ${ }^{4}$ are frequently prescribed, as is furosemide. ${ }^{7}$ A physician may also perform serial lumbar punctures to decrease CSF pressure.

Patients whose conditions cannot be managed with these methods, or whose vision is failing, have surgical options, including CSF shunting and optic nerve sheath fenestration (ONSF). ${ }^{8}$ However, these treatment options have some risks. Shunting can be highly effective, but the shunts often need to be revised or replaced, especially for obese patients. ${ }^{9}$ Whereas many patients experience relief from headaches with shunts, Rosenberg et $\mathrm{al}^{10}$ found that only 14 of 37 patients remained "cured" after a single 
surgical procedure. Physicians may use ONSF to preserve or stabilize a patient's vision, but the procedure often does not alleviate headache symptoms. ${ }^{11}$

We report a case of IIH that demonstrates the effects of digoxin, a medication that may benefit patients for whom other treatments have failed or who are unable or unwilling to undergo surgical procedures.

\section{Report of Case}

A 40-year-old woman presented to our neurology clinic in August 2007 with worsening headaches, which she had been having for several years, and decreased vision. She was legally blind in 1 eye and losing vision in the other eye. Her medical history was notable for IIH, common variable immunodeficiency, psoriatic arthritis, psoriasis, asthma, obesity (body mass index, 62), hypothyroidism, esophageal reflux, irritable bowel syndrome, nephrolithiasis, sinus tachycardia, osteoporosis, legionnaires' disease, recurrent respiratory infections, urinary tract infections, and sleep apnea. She managed her headaches with topiramate, $25 \mathrm{mg} 3$ times daily, and with therapeutic lumbar punctures, which she received from her previous neurologist as needed.

Aside from the worsening headaches and vision, she denied other signs or symptoms such as a change in weight, fevers, chills, night sweats, nausea, vomiting, chest pain, dyspnea, incontinence, or abdominal pain. She did not smoke tobacco or drink alcohol. Her family history revealed her father had psoriasis and died of esophageal cancer, and her mother had osteoarthritis of the hips.

The patient's medications included topiramate, triamcinolone cream, alclometasone dipropionate ointment, omeprazole, loperamide, alprazolam, methotrexate, etanercept, fluticasone inhaler, tiotropium bromide inhaler, beclometasone inhaler, cetirizine, meloxicam, levothyroxine, tegaserod, folate, verapamil, azelastine nasal spray, levalbuterol, calcitonin, and oxycodone/acetaminophen $5 / 325$ as needed for back pain.

Additionally, she had allergies to several medications, including acetazolamide, furosemide, ciprofloxacin, clarithromycin, sulfonylureas, salicylates, moxifloxacin, azithromycin, gatifloxacin, codeine, ceftibuten, naproxen, alendronate, erythromycin, and ceftriaxone.
During physical examination, the patient was alert and oriented to person, place, and time. She was able to follow simple commands, displayed abstract reasoning, and showed no aphasia. The cranial nerve examination showed loss of peripheral vision, bilaterally, with greater deficit in the left eye. The margins of her optic discs were not well defined. The results of the rest of her physical examination were unremarkable. A recent lumbar puncture had an opening pressure of $28 \mathrm{~cm} \mathrm{H}_{2} 0$. Findings of magnetic resonance images and magnetic resonance venograms of the brain were unremarkable.

We discussed the need for a change in treatment because of her vision problems and worsening headaches. Finding an appropriate medication was a challenge because of her allergies to acetazolamide and furosemide. She had been trying to lose weight for years but had also been taking steroids, which can cause weight gain, for her rheumatologic disorders. We presented the options of a CSF shunt or ONSF. However, the patient refused all surgical procedures because of her immunodeficiency, use of immunosuppressants, and problems with recurrent respiratory infections and urinary tract infections. She did not want to put herself at risk of infection or possible failure of a procedure.

Neblett et $\mathrm{al}^{12}$ suggested that digoxin can be beneficial to patients with IIH. We discussed this option with the patient and with cardiologists and cardiology staff. The patient agreed to try digoxin. After 6 months of treatment at a dosage of $0.125 \mathrm{mg}$ daily (in tablet form), her headaches disappeared and her vision stabilized. Of note, although the patient was unable to lose weight in the past, her weight decreased from $299 \mathrm{lb}$ to $285 \mathrm{lb}$ in the 6 months after starting digoxin.

The patient had been headache free for 2 years until she was hospitalized for an unrelated condition in the summer of 2009. During her hospitalization she was unable to take digoxin for 3 days, at which time her headaches came back, necessitating a lumbar puncture. After hospitalization, the patient resumed digoxin at the same dosage but in oral solution form $(0.05 \mathrm{mg} / \mathrm{mL}, 2.5 \mathrm{~mL}$ daily). In the past 4 years, she has had 1 flare-up of her $\mathrm{IIH}$, which was mild in comparison to her other flare-ups. Overall, her headaches have almost disappeared and her loss of vision has stabilized. 


\section{Comment}

There are multiple efficacious treatment options for patients with IIH. However, when a patient presents with a complicated medical history, treatment becomes more challenging. The patient was not benefitting from standard medical therapy, and an intervention was needed to preserve her vision and manage her headaches. Although we could not isolate a cause for the patient's headaches, we believed that nonintervention would adversely affect the patient.

Few cases of IIH managed with digoxin have been reported, to our knowledge. Digoxin acts on the ouabainsensitive $\mathrm{Na}^{+} / \mathrm{K}^{+}$ATPase receptors in the choroid plexus, thereby decreasing CSF production. Neblett et al ${ }^{12}$ discussed 3 cases in which the use of digoxin correlated with decreasing CSF production by up to $78 \%$. They concluded that digoxin may be beneficial to conditions of increased CSF pressure. Schott and Holt, ${ }^{13}$ however, used digoxin in a patient with IIH without success. This instance may illustrate that there is more than 1 mechanism for the increased intracranial pressure and perhaps the mechanism in our patient is different from the mechanism in the patient noted by Schott and Holt. ${ }^{13}$ Borsody et $\mathrm{al}^{14}$ noted that levels of brain ouabain-like compounds, which inhibit the $\mathrm{Na}^{+} / \mathrm{K}^{+}$ATPase receptors, were lower in patients with unsuccessfully managed IIH. Digoxin is similar to ouabain-like compounds, which could explain our patient's improvement.

The patient lost $14 \mathrm{lb}$ in the first 6 months of treatment, a result that correlates with that of Hannerz, ${ }^{6}$ who found that treatment with digoxin caused weight loss and a decrease in CSF pressure in obese patients. Although our patient continued to lose weight $(78 \mathrm{lb}$ during the course of 4 years), she also experienced some gastrointestinal motility problems during the same time span. Therefore, it cannot be said whether digoxin is responsible for the patient's weight loss.

\section{Conclusion}

In patients with IIH and complicated medical conditions, physicians should consider digoxin. Even though the mechanism of action is not well understood, it clearly has benefits in this group of patients.

\section{References}

1. Friedman DI, Gordon LK, Egan RA, et al. Doxycycline and intracranial hypertension. Neurology. 2004;62(12):2297-2299.

2. Friedman DI, Jacobson DM. Diagnostic criteria for idiopathic intracranial hypertension. Neurology. 2002;59(10):1492-1495.

3. Radhakrishnan K, Ahlskog JE, Cross SA, Kurland LT, O'Fallon WM. Idiopathic intracranial hypertension (pseudotumor cerebri): descriptive epidemiology in Rochester, Minn, 1976 to 1990. Arch Neurol. 1993;50(1):78-80.

4. Binder DK, Horton JC, Lawton MT, McDermott MW. Idiopathic intracranial hypertension. Neurosurgery. 2004;54(3):538-552. doi:10.1227/01.NEU.0000109042.87246.3C.

5. Biousse V, Bruce BB, Newman NJ. Update on the pathophysiology and management of idiopathic intracranial hypertension [published online March 15, 2012]. J Neurol Neurosurg Psychiatry. 2012;83(5):488-494. doi:10.1136/jnnp-2011-302029.

6. Hannerz J. Decrease of intracranial pressure and weight with digoxin in obesity. J Clin Pharmacol. 2001;41(4):465-468.

7. Liu GT, Glaser JS, Schatz NJ. High-dose methylprednisolone and acetazolamide for visual loss in pseudotumor cerebri. Am J Opthalmol. 1994;118(1):88-96.

8. Wall M, George D. Idiopathic intracranial hypertension: a prospective study of 50 patients. Brain. 1991;114(pt 1A):155-180.

9. Burgett RA, Purvin VA, Kawasaki A. Lumboperitoneal shunting for pseudotumor cerebri. Neurology. 1997;49(3):734-739.

10. Rosenberg ML, Corbett JJ, Smith C, et al. Cerebrospinal fluid diversion procedures in pseudotumor cerebri. Neurology. 1993;43(6):1071-1072.

11. Banta JT, Farris BK. Pseudotumor cerebri and optic nerve sheath decompression. Ophthalmology. 2001;107(10):1907-1912.

12. Neblett CR, Waltz TA Jr, McNeel DP, Harrison GM. Effect of cardiac glycosides on human cerebrospinal-fluid production. Lancet. 1972;2(7785):1008-1009.

13. Schott GD, Holt D. Digoxin in benign intracranial hypertension [letter]. Lancet. 1974;1(7853):312-313.

14. Borsody M, Semenov I, Carroll K, et al. The relation of brain ouabain-like compounds and idiopathic intracranial hypertension. Headache. 2006;46(8):1255-1260. doi:10.1111/j.1526-4610.2006.00527.x.

() 2014 American Osteopathic Association 